\title{
Six-month bracket failure rate with a flowable composite: A split-mouth randomized controlled trial
}

\author{
Sindhuja Krishnan'1, Saravana Pandian², R. Rajagopal
}

DOI: http://dx.doi.org/10.1590/2177-6709.22.2.069-076.oar

Introduction: The use of flowable composites as an orthodontic bonding adhesive merits great attention because of their adequate bond strength, ease of clinical handling and reduced number of steps in bonding. Objective: The aim of this Randomized Controlled Trial was to comparatively evaluate over a 6-month period the bond failure rate of a flowable composite (Heliosit Orthodontic, Ivoclar Vivadent AG, Schaan) and a conventional orthodontic bonding adhesive (Transbond XT, 3M Unitek). Methods: 53 consecutive patients (23 males and 30 females) who fulfilled the inclusion and exclusion criteria were included in the study. A total of 891 brackets were analyzed, where 444 brackets were bonded using Heliosit Orthodontic and 447 brackets were bonded using Transbond XT. The survival rates of brackets were estimated with the Kaplan-Meier analysis. Bracket survival distributions for bonding adhesives, tooth location and dental arch were compared with the log-rank test. Results: The failure rates of the Transbond XT and the Heliosit Orthodontic groups were $8.1 \%$ and $6 \%$ respectively. No significant differences in the survival rates were observed between them $(p=$ 0.242). There was no statistically significant difference in the bond failure rates when the clinical performance of the maxillary versus the mandibular arches and the anterior versus the posterior segments were compared. Conclusions: Both systems had clinically acceptable bond failure rates and are adequate for orthodontic bonding needs.

Keywords: Bond failure. Flowable composite. Orthodontic bonding. Bracket survival. Adhesive. Debonding rate.

Introdução: o uso de resinas compostas fluidas como agentes de cimentação em Ortodontia tem merecido grande atenção, em função de sua adequada capacidade adesiva, facilidade de uso clínico e número reduzido de etapas de colagem. Objetivo: o objetivo deste estudo randomizado controlado foi avaliar o índice de falhas nos 6 meses após a colagem com uma resina composta fluida (Heliosit Orthodontic, Ivoclar Vivadent AG, Schaan), em comparação com um adesivo ortodôntico convencional (Transbond XT, 3M Unitek). Métodos: 53 pacientes consecutivos (23 homens e 30 mulheres) que se enquadravam nos critérios de inclusão adotados foram incluídos no presente estudo. No total, 891 braquetes foram analisados, sendo 444 colados com o Heliosit Orthodontic e 447 colados com o Transbond XT. As taxas de sobrevivência dos braquetes foram estimadas por meio da análise de Kaplan-Meier. As distribuições das taxas de sobrevivência dos braquetes em função do adesivo usado, do dente e da arcada dentária em questão foram comparadas por meio do teste de log-rank. Resultados: os índices de falhas para os grupos Transbond XT e Heliosit Orthodontic foram, respectivamente, de 8,1\% e 6\%. Não foram observadas diferenças significativas entre os grupos quanto às taxas de sobrevivência dos braquetes $(p=0,242)$. Também não foram observadas diferenças estatisticamente significativas quanto aos índices de falhas quando se comparou a performance clínica nas arcadas dentárias superior e inferior, e nos segmentos anterior e posterior da boca. Conclusões: ambos os sistemas apresentaram índices de falhas clinicamente aceitáveis, podendo ser considerados adequados para a colagem ortodôntica.

Palavras-chave: Falha de colagem. Resina composta fluida. Colagem ortodôntica. Sobrevivência de braquete. Adesivo. Índice de falhas.

${ }^{1}$ Postgraduation program, Department of Orthodontics and Dentofacial Orthopaedics, Saveetha Dental College, Saveetha University, Chennai, Tamil Nadu, India.

${ }^{2}$ Senior Lecturer, Department of Orthodontics and Dentofacial Orthopaedics, Saveetha Dental College, Saveetha University, Chennai, Tamil Nadu, India.

${ }^{3}$ Professor, Department of Orthodontics and Dentofacial Orthopaedics, Saveetha Dental College, Saveetha University, Chennai, Tamil Nadu, India.

» The authors report no commercial, proprietary or financial interest in the products or companies described in this article.
How to cite this article: Krishnan S, Pandian S, Rajagopal R. Six-month bracket failure rate with a flowable composite: A split-mouth randomized controlled trial. Dental Press J Orthod. 2017 Mar-Apr;22(2):69-76. DOI: http://dx.doi.org/10.1590/2177-6709.22.2.069-072.oar

Submitted: July 19, 2016 - Revised and accepted: September 27, 2016

Contact address: Sindhuja Krishnan

Department of Orthodontics and Dentofacial Orthopaedics, Madha Dental College, MGR University, Chennai, Tamilnadu, India

E-mail: reachsindhuja@yahoo.com 


\section{INTRODUCTION}

In orthodontics, it's a standard clinical practice to bond brackets to etched teeth using chemical or light-curing adhesive systems. The high initial bond strength, the minimal extent of oxygen inhibition and the extended working time for optimal bracket placement have contributed to the popularity of light-curing adhesives. ${ }^{1,2,3}$

The majority of adhesives currently used for orthodontic bonding are complex materials composed of synthetic polymers such as bisphenol-A glycol dimethacrylate (Bis-GMA) with either ethylene glycol dimethacrylate (EGDMA) or triethylene glycol dimethacrylate (TEGDMA) as a diluent to reduce the viscosity of Bis-GMA. In addition to the above, molecules that promote or modify the polymerization reaction are incorporated. ${ }^{4}$

Filler particles are incorporated into a resin matrix to improve its mechanical properties.

The primary purpose of the filler particles is to increase the strength of the composite and reduce the amount of matrix material. The fillers provide reinforcement of matrix material, reduction in polymerization shrinkage and reduction in thermal expansion and contraction (dimensional changes). It results in reduced microleakage, improved workability with increased viscosity, reduction in water sorption and softening. ${ }^{5,6,7}$

Among the composite resins that could be used as orthodontic adhesives, flowable composites merit great attention due to their clinical handling characteristics. ${ }^{8}$ Flowable composites exhibit two desirable clinical handling characteristics that have not existed for composites until very recently: ${ }^{9}$

1) no stickiness;

2) fluid injectability.

The other desirable characteristics include adequate bond strength, sufficient working time, shorter curing time, and improved ease of use.

Flowable composites retain the same small particle sizes of traditional hybrid composites, but have less filler content, thus, reducing the viscosity of the mixture. Heliosit Orthodontic (Ivoclar Vivadent AG, Schaan) is a light-curing, highly translucent single-component bonding material for brackets and is supplied in convenient syringes. The monomer matrix consists of urethane dimethacrylate, Bis-GMA and decandiol dimethacrylate $(85 \mathrm{wt} \%)$. The filler consists of a highly dispersed silicon dioxide (14wt\%). The additional contents are catalysts and stabilizers $(1 \mathrm{wt} \%) .{ }^{10}$ Heliosit Orthodontic was developed to ease the bonding procedure of orthodontic attachments by eliminating the need for primer application both on the bracket base and on etched tooth surface.

The purpose of this randomized clinical trial was to compare the bond failure rates of brackets bonded with a flowable composite (Heliosit Orthodontic, Vivadent Ivoclar, Schaan) and a conventional multi-step system (Transbond XT, 3M Unitek, Ca, USA) over a 6-month period. The secondary aim was to investigate factors contributing to bracket failure, as tooth location and dental arch. The null hypothesis is that there is no difference in the failure rates of brackets bonded with Heliosit Orthodontic or Transbond XT during the first 6 months of preadjusted edgewise appliance therapy.

\section{MATERIAL AND METHODS \\ Estimation of power and sample size}

The sample size of the study was estimated by the number of brackets bonded either with Transbond XT or Heliosit Orthodontic. In this study, each bonded bracket was the unit of measurement. To obtain an adequate power of $80 \%$, the sample size was determined to be 813 brackets. For 813 brackets, approximately 53 patients were required. A buffer of $20 \%$ was included in order to compensate for any loss of patients during follow-up. Institutional review board clearance and approval from human ethical committee were obtained from the Saveetha University for this single-centered study and patients gave their written consent for participation.

\section{Inclusion criteria}

" Age group: 13 to 30 years.

" Complete permanent dentition.

" Patient requiring fixed appliance therapy in both arches.

"Both extraction and non-extraction cases.

" Absence of labial or buccal restoration.

\section{Exclusion criteria}

" Patient with congenital enamel defects, fillings or hypoplasia.

" Partially erupted teeth.

» Second and third molars. 
"Surgically exposed teeth.

"Dentition with occlusal interferences.

Thus, 53 consecutive patients $(23$ males and 30 females) who fulfilled the inclusion criteria were selected for this study. Patients were selected from those seeking orthodontic treatment at the Orthodontics Department of Saveetha University.

\section{Study design}

The study design was a single blinded, split-mouth, cross-arch Randomized Controlled Trial. Transbond XT was used as the control material and Heliosit Orthodontic was used as the experimental material.

The patients were unaware of the material and the side of the mouth chosen for bonding of the brackets. The decision as to the choice of material for each side was allocated by using a Random Number Table. The adhesives were separated based on group category. In Group A, the brackets were bonded with Transbond XT on the right side and Heliosit Orthodontic on the left side of maxillary and mandibular arches, and vice versa in Group B (Table 1). This was done so that both materials would be equally distributed on maxillary and mandibular right and left quadrants.

The total number of patients involved in the study was 53 (23 males and 30 females). For 53 patients, the total number of brackets bonded was 901 . All the patients were bonded with 0.022-in slot MBT prescription (Gemini stainless steel brackets, 3M Unitek). Two patients were excluded from the study as they did not come regularly for the monthly follow-up. Another patient discontinued treatment after the third month of bonding. By the end of the 6-month trial, the total number of patients analyzed was 50 and the number of brackets analyzed was 891 . The characteristics of the study sample are described in Table 2.

\section{Bonding procedure}

It was not possible to blind the operators to the bonding system being used because the two systems had different forms of application. The teeth were cleaned using a rubber cup with pumice and water slurry, rinsed, isolated with cheek retractors and a low-volume suction evacuator, and dried with oilfree air. For the teeth to be bonded using Transbond $\mathrm{XT}, 37 \%$ phosphoric acid was applied to the enamel surface for 15 seconds before rinsing with water
Table 1 - Quadrant-wise distribution of adhesive

\begin{tabular}{ccc}
\hline GROUPS & Right side & Left side \\
A & Transbond XT & Heliosit Orthodontic \\
B & Heliosit Orthodontic & Transbond XT \\
\hline
\end{tabular}

Table 2 - Sample characteristics.

\begin{tabular}{|ccc|}
\hline & $n$ & $\%$ \\
\hline Number of patients & 53 & - \\
\hline Number of brackets & 891 & - \\
\hline Distribution of brackets by bonding material & \\
\hline Transbond XT & 447 & $50.1 \%$ \\
\hline Heliosit Orthodontic & 444 & $49.9 \%$ \\
\hline Distribution of brackets by dental arches & \\
\hline Maxillary & 445 & $49.9 \%$ \\
\hline Mandibular & 446 & $50.1 \%$ \\
\hline Distribution of brackets by tooth type & \\
\hline Anterior & 601 & $67.45 \%$ \\
\hline Posterior & 290 & $32.55 \%$ \\
\hline Distribution of brackets by side of the arches & \\
\hline Right side & 438 & $49.15 \%$ \\
\hline Left side & 453 & $50.85 \%$ \\
\hline
\end{tabular}

and drying until the enamel became frosty white. Transbond XT primer was then applied to the etched enamel according to the manufacturer's instructions and given a gentle burst of air. Transbond XT adhesive paste was placed on the back of the brackets.

For the teeth to be bonded using Heliosit Orthodontic, 37\% phosphoric acid was applied to the enamel surface for 15 seconds before rinsing with water and drying until the enamel was frosty white. As per the manufacturer's recommendation no intermediate primer was applied on the surface to be bonded. Then Heliosit Orthodontic was placed on the back of the brackets and the brackets were placed onto the etched enamel surface.

In both groups the brackets were positioned along the long axis of the teeth with the help of a bracket positioning gauge, according to the MBT bracket positioning chart. Sufficient pressure was applied to squeeze out excess adhesive, which was removed from the margins of the bracket 
base with an explorer before polymerization. When satisfied with the bracket positioning, the adhesive was cured using a Halogen light curing unit (QHL75, Dentsply). The adhesive was cured from the occlusal, gingival, mesial and distal aspects for 10 seconds each.

Standardization was achieved by bonding all the brackets in one appointment by the same operator. Aligning archwire of choice was either a 0.014-in NiTi wire or a 0.016 -in NiTi wire, depending on the initial degree of alignment and crowding. These wires were tied approximately 10 minutes after bonding, using elastomeric modules. No bite planes were used during treatment. The study was concluded before the addition of edgewise archwires. Verbal and written instructions about diet and care were given immediately to the patient after fitting the appliances.

\section{Observation and follow-up}

The bonding, follow-up and assessment of bond failure rate of the brackets was done by a single operator (investigator). Patients were followed for a period of 6 months. If a bond failed, the following information was recorded: (1) site of bond failure, (2) number of failed brackets (3) date of bond failure, and (4) possible reason for bond failure. The patients were treated at 3-4 week intervals but were requested to come as soon as possible in case of a bond failure. When the patient was unaware of a bracket failure, the date of the appointment was recorded as the date of failure. Based on the tooth location, they were divided into anterior and posterior segments. The first and second premolars brackets were evaluated in the posterior segment as the molars were banded. Failed brackets were rebonded with the conventional adhesive, but not included in the further study.

\section{Statistical analysis}

Statistical data analysis was carried out by using the software SPSS v. 4.0 (SPSS Inc., Chicago, USA), at the $5 \%$ level of significance. The survival rates of the brackets were estimated by using the Kaplan-Meier test. Kaplan-Meier estimates of bracket survival curves were plotted. Bracket survival distributions with respect to bonding procedure, dental arch (maxillary and mandibular) and tooth location (anterior and posterior) were compared using the log-rank test $(p<0.05)$.

\section{RESULTS}

The flow chart of the trial is given in Figure 1. During the 6-month observation period, 63 (7.1\%) brackets failed: $27(6 \%)$ in the Heliosit Orthodontic group and 36 (8.1\%) in the Transbond XT group (Table 3). The corresponding bracket survival curves were plotted by using the KaplanMeier product-limit estimate (Fig 2). There was no significant difference in terms of bracket failure risk over the 6 months between groups ( $p=0.242$, hazard ratio $=0.69 ; 95 \%$ confidence interval 0.35-1.40; $\log$ rank test $P=0.251$ ).

The maxillary arches had a $3.3 \%$ failure rate, and the mandibular arches a 3.8\% failure rate; these were not statistically significant according to the log-rank test $(P=0.518$; hazard ratio $=0.71 ; 95 \%$ confidence level $=0.36-1.43)$. The influence of the dental arches on bracket survival rate is shown in Fig 3.

Posterior brackets (premolars) showed lesser (2.6\%) failure rates than anterior brackets (4.5\%). Figure 4 shows the influence of tooth location on bracket survival rate. The log-rank test showed no significant differences between anterior and posterior brackets in terms of survival rate $(P=0.0492$; hazard ratio $=0.42$; $95 \%$ confidence level $=0.20-0.83$.

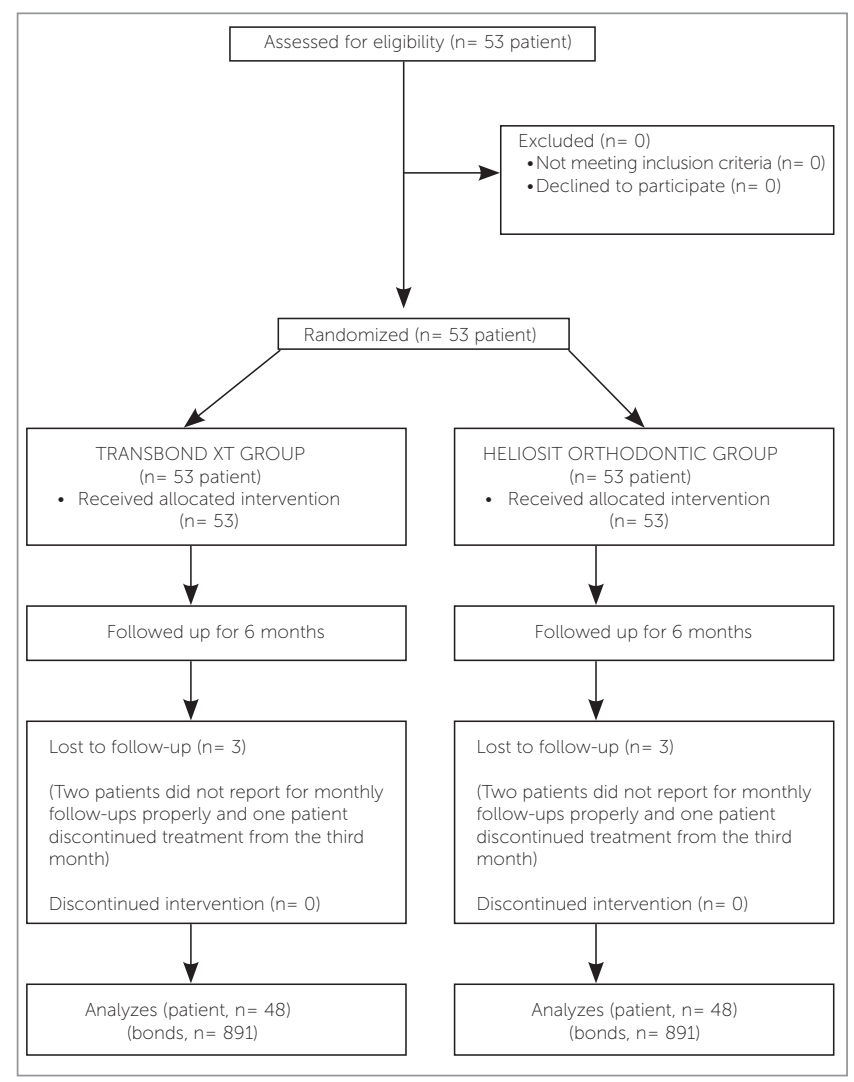

Figure 1 - The CONSORT flow diagram of the trial. 


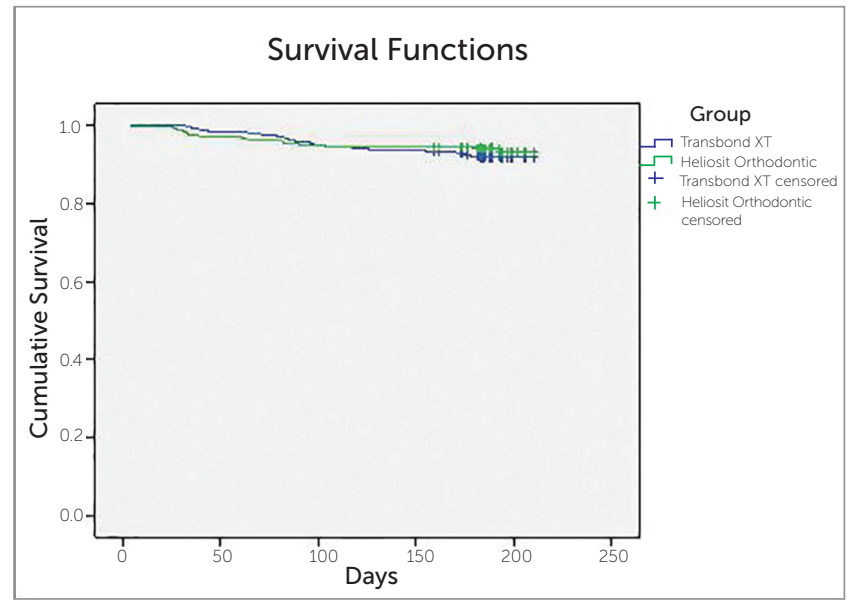

Figure 2 - Overall Kaplan-Meier survival plot comparing bond failure between Transbond XT (3M Unitek) and Heliosit Orthodontic (Ivoclar Vivadent).

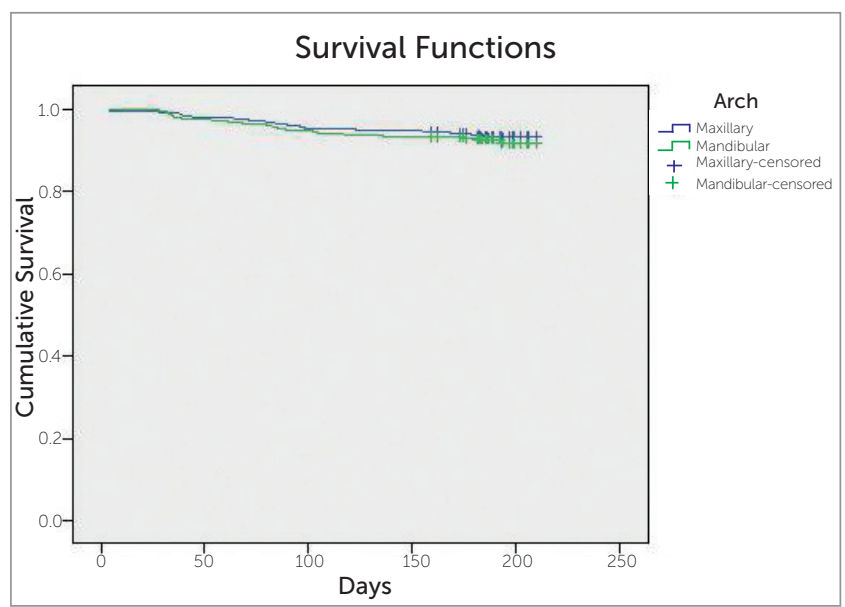

Figure 3 - Overall Kaplan-Meier survival plot comparing bond failure in maxillary and mandibular arches.

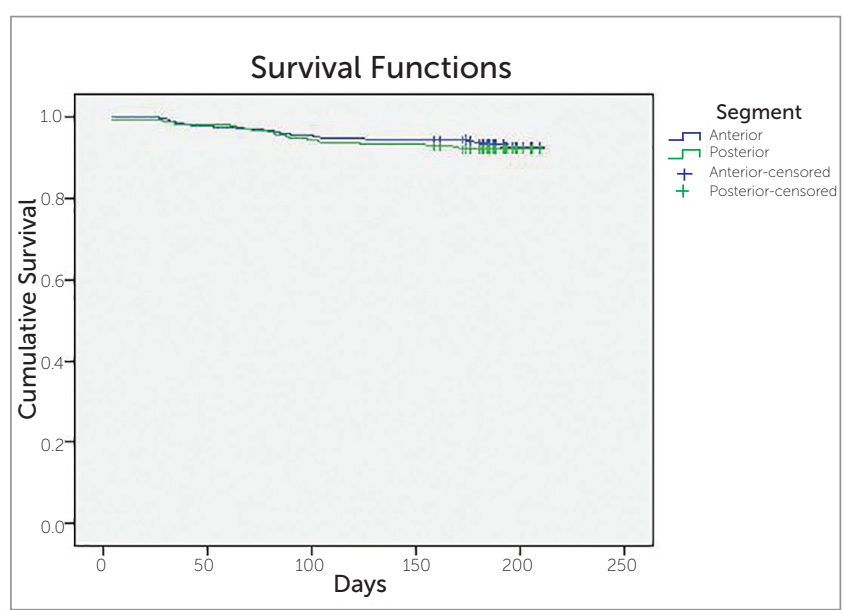

Figure 4 - Overall Kaplan-Meier survival plot comparing bond failure in anterior and posterior segments.

\section{DISCUSSION}

There was no statistically significant difference in bond failure rates between the Transbond XT and the Heliosit Orthodontic groups at $p<0.05$. The overall bond failure in this study was 63 brackets with $7.1 \%$ failure rate. The bond failure of Transbond XT and Heliosit Orthodontic was 36 (8.1\%) and 27 (6.0\%), respectively (Table 3 ). Studies by O'Brien et al, ${ }^{11}$ Adolfsson et $\mathrm{al}^{12}$ and Cal-Neto et $\mathrm{a}^{13}$ reported failure rates of $4.7-6.6 \%, 7.2 \%$ and $5.48 \%$, respectively, for various adhesive-bracket combinations.

Bond failure rates below 10\% are generally considered clinically acceptable. ${ }^{14}$ It is difficult to make a direct comparison between studies due to the variety of techniques, materials, research designs and trial durations. ${ }^{15}$

In any time scale, the overall failure rates for a clinical sample can be calculated. This could provide a straightforward statement of the overall percentage of failures in a sample over a certain time, or it can be used to compare variables in a sample. Failure rates are a widely accepted means of representing the performance of brackets. ${ }^{16}$

In in vivo studies, socioeconomic and dental status of patients, malocclusion classification and resultant mechanotherapy may affect the outcomes. Furthermore, masticatory forces varying with facial type, culturally influenced dietary habits, and sex differences may also influence the results. ${ }^{17}$

Heliosit Orthodontic was developed to facilitate orthodontic bonding by eliminating the need for primer application both on the bracket base and the etched tooth surface. It is a Bis-GMA-based lightcuring orthodontic adhesive designed for bonding ceramic and metal orthodontic brackets. Its monomer matrix consists of urethane dimethacrylate, Bis-GMA and decandiol dimethacrylate (85 wt\%). The filler consists of highly dispersed silicon dioxide (14 wt\%). Additional contents are catalysts and stabilizers (1 wt\%). Although Heliosit Orthodontic was initially developed for bonding of brackets, its application has been as an adhesive for bonding lingual retainers, ${ }^{28,29}$ and even as a luting cement for prosthesis. ${ }^{18}$

Heliosit Orthodontic has not been widely studied to clinically assess its bonding efficacy. Reynolds ${ }^{19}$ stated that bond strengths of 5.9 to $7.8 \mathrm{MPa}$ were clinically acceptable and Heliosit Orthodontic adhesive had shear bond strength within the already-mentioned clinically 
Table 3 - Overall bracket failure rate between Heliosit Orthodontic (Ivoclar Vivadent, Schaan, Liechtenstein) and Transbond XT (3M Unitek, Ca, USA).

\begin{tabular}{|c|c|c|c|c|c|}
\hline Variable & Number & Bracket failures & Failure rate (\%) & p value & log-rank \\
\hline \multicolumn{6}{|c|}{ Material used for bonding procedure } \\
\hline Transbond XT & 444 & 36 & $8.1 \%$ & \multirow{2}{*}{0.242} & \multirow{2}{*}{.251} \\
\hline Heliosit Orthodontic & 447 & 27 & $6 \%$ & & \\
\hline \multicolumn{6}{|c|}{ Dental arch } \\
\hline Maxillary arch & 445 & 29 & $3.3 \%$ & \multirow{2}{*}{0.601} & \multirow{2}{*}{.518} \\
\hline Mandibular arch & 446 & 34 & $3.8 \%$ & & \\
\hline \multicolumn{6}{|c|}{ Bracket location } \\
\hline Anterior region & 601 & 40 & $4.5 \%$ & \multirow{2}{*}{0.488} & \multirow{2}{*}{.492} \\
\hline Posterior region & 290 & 23 & $2.6 \%$ & & \\
\hline
\end{tabular}

acceptable range. Few in vitro studies have been carried out to evaluate its bond strength. While comparing Heliosit Orthodontic with Transbond XT, studies concluded that Transbond XT exhibited higher bond strengths in all the studies. However, the bond strengths of Heliosit Orthodontic were clinically acceptable. ${ }^{20,21,22}$ Manufacturers claim that its flexural strength is $80 \mathrm{MPa}$ and that the shear bond strength of brackets on etched enamel is $10 \mathrm{MPa}$, for ceramic brackets and $12 \mathrm{MPa}$ for metal brackets.

Unlike orthodontic bonding systems, such as Transbond XT, Heliosit Orthodontic can be applied to acidetched enamel without the use of intermediate bonding resin due to its low filler loading and improved flowability. By reducing the number of steps during bonding, clinicians can save time and reduce potential errors related to contamination during the bonding procedure. It also allows easier and more even application to the mesh base of the brackets. Another added advantage of using this flowable composite is that it proves to be more cost efficient as it does not require an intermediate primer. Thus, as Heliosit Orthodontic guarantees clinically acceptable survival rate, it is undoubtedly advantageous for orthodontic bracket bonding.

Since not all brackets failed by the end of the study period, a survival analysis was done. This analysis differs from other types of statistics because it can use partial or censored information. This analysis was used here because non-failed attachments were all censored at the conclusion of the treatment, and it is impossible to follow all the brackets to failure. The Kaplan-Meier survival analysis showed that the mean survival time for brackets bonded with Transbond XT (178.83 days) was similar to Heliosit Orthodontic (179.03 days) (Fig 2).

Another factor observed in relation to survival time was that, in the present study, the maximum number of bond failures occurred during the initial 3 months of treatment. The most common reasons cited by the patients for the bond failures were hard brushing and biting on a hard food substances.

O'Brien et $\mathrm{al}^{11}$ presented three possible reasons for the increased failure rate during the first 6 months of treatment:

1) They suggested that any deficiencies in the bond strength of any individual bracket/adhesive combination would become evident within this initial period of treatment.

2) The initial period of treatment is also a time of acclimatization and experimentation for patients concerning the type of food that can be tolerated by fixed orthodontic appliances.

3) The initial phase of treatment may involve a period of overbite correction and, therefore, heavy occlusal forces may be applied to many of the bonded attachments.

In the present study, failure rates demonstrated no significant differences between maxillary and mandibular brackets, with mandibular bonds failing more frequently (maxillary $=3.3 \%$ bond failure; mandibular $=3.8 \%$ bond failure) at $p=0.304$ (Table 3). Similar results have 
been obtained in other studies which found that the failure rate of maxillary brackets was less than the failure rate of mandibular brackets. ${ }^{23-26}$

Potential reasons for this could include increased masticatory load on mandibular brackets. In patients with a normal transverse arch relationship, brackets bonded to mandibular teeth have potential antagonists in centric relation, whereas maxillary brackets do not.

It is evident that posterior teeth failed less frequently than anterior teeth. This was true for the overall sample (posterior $=2.6 \%$ bond failure and mean survival time of 178.33 days; anterior $=4.5 \%$ bond failure and mean survival time of 179.22 days). However, the findings were not statistically significant at $p<0.05$ (Table 3 ).

Many studies report that posterior teeth suffer more bracket failures than do brackets on anterior teeth. The higher occlusal forces on posterior teeth, the difficulty of access and moisture control and the more aprismatic enamel on premolars could be possible reasons for this scenario. ${ }^{11,27}$

The literature on bonding has shown that the pattern of orthodontic bond failure in vivo is not uniform for all the teeth in either dental arch and also between the arches. This occurs even though all the teeth are bonded by the same operator using the same adhesive and a standard protocol, thus emphasizing that only certain sites in the mouth have a greater predilection for failures than others. This can be due to some factors within the oral cavity like tooth morphology, masticatory forces and chewing pattern, which predispose certain sites to a greater rate of bond failure.

Present results indicate that both Transbond XT and Heliosit Orthodontic can be efficiently used for bonding orthodontic appliances. From a clinical standpoint, the use of Heliosit Orthodontic can be more advantageous because it reduces the number of clinical steps required to bond brackets and, thus, saves chair time. It is also cost effective. Undoubtedly, it improves the quality of bonding and efficiency of the operator by reducing the risk of salivary contamination during the bonding procedure. A disadvantage to this technique of using flowable composites as orthodontic bonding adhesive is that it denies application of a filled sealant that protects the enamel from white spot lesions.

However, the choice of a particular orthodontic bonding adhesive will depend on the clinical preference of the operator.

\section{CONCLUSIONS}

1) In this randomized controlled trial, the conventional adhesive (Transbond XT) and the flowable composite (Heliosit Orthodontic) had similar and clinically acceptable bond failure rates.

2) Heliosit Orthodontic is a more desirable composite because it reduces the number of clinical steps. It reduces chair time, is cost effective and reduces the risk of salivary contamination.

\section{Formatting of funding sources}

This research did not receive any specific grant from funding agencies in the public, commercial, or not-for-profit sectors. 


\section{REFERENCES}

1. Buyukyilmaz T, Usumez S, Karaman Al. Effect of self-etching primers on bond strength-are they reliable? Angle Orthod. 2003 Feb;73(1):64-70

2. Eliades T, Eliades G, Brantley WA, Johnston WM. Polymerization efficiency of chemically cured and visible light-cured orthodontic adhesives: degree of cure. Am J Orthod Dentofacial Orthop. 1995 Sept;108(3):294-301.

3. Uysal T, Basciftci FA, Uşümez S, Sari Z, Buyukerkmen A. Can previously bleached teeth be bonded safely? Am J Orthod Dentofacial Orthop. 2003 June:123(6):628-32

4. Faltermeier A, Rosentritt M, Faltermeier R, Reicheneder C, Müssig D Influence of filler level on the bond strength of orthodontic adhesives. Angle Orthod. 2007 May:77(3):494-8.

5. Brantley WA, Eliades T. Orthodontic materials. Scientific and clinical aspects. New York: Thieme; 2001

6. Bishara SE, Aljouni R. Evaluation of the orthodontic application of two new restorative systems. Hellenic Orthod Review. 2004;(7):25-32.

7. Anusavice K, Shen C, Rawls R. Phillips Science of Dental Materials. 12th ed. St. Louis: Saunders; 2012

8. Elaut J, Asscherickx K Vande Vannet B, Wehrbein H. Flowable composites for bonding lingual retainers. J Clin Orthod. 2002 Oct:36(10):597-8

9. Tecco S, Traini T, Caputi S, Festa F, de Luca V, D'Attilio M. A new one-step dental flowable composite for orthodontic use: an in vitro bond strength study. Angle Orthod. 2005 July:75(4):672-7.

10. Ivoclar Vivadent. Heliosit Orthodontic product profile. Liechtenstein Ivoclar Vivadent; 2003.

11. O'Brien KD, Read MJ, Sandison RJ, Roberts CT. A visible light-activated direct-bonding material: an in vivo comparative study. Am J Orthod Dentofacial Orthop. 1989 Apr:95(4):348-51.

12. Adolfsson $U$, Larsson E, Øgaard B. Bond failure of a no-mix adhesive during orthodontic treatment. Am J Orthod Dentofacial Orthop. 2002 Sept;122(3):277-81

13. Cal-Neto JP, Miguel JA, Zanella E. Effect of a self-etching primer on shear bond strength of adhesive precoated brackets in vivo. Angle Orthod 2006 Jan:76(1):127-31

14. Mavropoulos A, Karamouzos A, Kolokithas G, Athanasiou AE. In vivo evaluation of two new moisture-resistant orthodonticadhesive systems: a comparative clinical trial. J Orthod. 2003 June:30(2):139-47; discussion 127-8.
15. Littlewood SJ. Investigation of a hydrophilic primer for orthodontic bonding: an in vitro study. J Orthod. 2000 June:27(2):181-6

16. Millett DT. A comparative clinical trial of a compomer and a resin adhesive for orthodontic bonding. Angle Orthod. 2000 June:70(3):233-40

17. Pandis N, Theodore Eliades. A comparative in vivo assessment of the long term failure rate of 2 self etching primers. Am J Orthod Dentofacial Orthop. 2005:128(1):96-8

18. Breeding LC, Dixon DL, Caughman WF. The curing potential the curing potential of light-activated composite resin luting agents. J Prosthet Dent. 1991 Apr;65(4):512-8

19. Reynolds IR. A review of direct orthodontic bonding. Br J Orthod $1975: 2(3): 171-8$

20. Murray SD, Hobson RS. Comparison of in vivo and in vitro shear bond strength. Am J Orthod Dentofacial Orthop. 2003 Jan;123(1):2-9.

21. Durrani OK. In vitro comparison of shear bond strength of Transbond XT and Heliosit Orthodontic as Direct bracket bonding adhesives. Pakistan Oral Dent J. 2008:28(2):203-6

22. Bradburn G, Pender N. An in vitro study of the bond strength of two lightcured composites used in the direct bonding of orthodontic brackets to molars. Am J Orthod Dentofacial Orthop. 1992 Nov;102(5):418-26.

23. Newman GV. Epoxy adhesives for orthodontic attachments: progress report. Am J Orthod Dentofacial Orthop. 1965;51(12):901-12

24. Zachrisson BU, Brobakken BO. Clinical comparison of direct versus indirect bonding with different bracket types and adhesives. Am J Orthod. 1978 July:74(1):62-78

25. Trimpeneers LM, Dermaut LR. A clinical trial comparing the failure rates of two orthodontic bonding systems. Am J Orthod Dentofacial Orthop. 1996:110(5):547-50

26. Linklater RA. Bond failure patterns in vivo. Am J Orthod Dentofacial Orthop. 2003 May:123(5):534-9

27. Sunna S, Rock WP. Clinical performance of orthodontic brackets and adhesive systems: a randomized clinical trial. Br J Orthod. 1998 Nov:25(4):283-7

28. Radlanski RJ, Zain ND. Stability of the bonded lingual wire retainer-a study of the initial bond strength. J Orofac Orthop. 2004;65:321-35

29. Stormann I, Ehmer U. A prospective randomized study of different retainer types. J Orofac Orthop. 2002:63:42-50. 